Commun. math. Phys. 6, 286-311 (1967)

\title{
Nonrelativistic Particles and Wave Equations
}

\author{
Jean-Marc Lévy-Leblond* \\ Laboratoire de Physique Théorique, Faculté des Sciences de Nice
}

Received April 1, 1967

\begin{abstract}
This paper is devoted to a detailed study of nonrelativistic particles and their properties, as described by Galilei invariant wave equations, in order to obtain a precise distinction between the specifically relativistic properties of elementary quantum mechanical systems and those which are also shared by nonrelativistic systems. After having emphasized that spin, for instance, is not such a specifically relativistic effect, we construct wave equations for nonrelativistic particles with any spin. Our derivation is based upon the theory of representations of the Galilei group, which define nonrelativistic particles. We particularly study the spin 1/2 case where we introduce a four-component wave equation, the nonrelativistic analogue of the Dirac equation. It leads to the conclusion that the spin magnetic moment, with its Landé factor $g=2$, is not a relativistic property. More generally, nonrelativistic particles seem to possess intrinsic moments with the same values as their relativistic counterparts, but are found to possess no higher electromagnetic multipole moments. Studying "galilean electromagnetism" (i.e. the theory of spin 1 massless particles), we show that only the displacement current is responsible for the breakdown of galilean invariance in Maxwell equations, and we make some comments about such a "nonrelativistic electromagnetism". Comparing the connection between wave equations and the invariance group in both the relativistic and the nonrelativistic case, we are finally led to some vexing questions about the very concept of wave equations.
\end{abstract}

\section{Introduction}

The subject of this paper ${ }^{1}$ may well seem doubly obsolete: is it really worth while investigating wave equations in nonrelativistic situations, when the battle-front of theoretical physics today runs through Quantum Field Theory (and/or S-matrix Theory) in completely relativistic contexts?

We think that the main justification to the present work lies in some of the peculier results which we obtain; as a matter of fact, these results show the necessity of revising certain commonly held ideas on quantum mechanics (QM). Specifically, it is most important, when one goes from nonrelativistic quantum mechanics (NQM) to relativistic quantum mechanics (RQM) to fully appreciate and clearly point out what are the new features brought into the theory by the change in the underlying kinematical group, as well as the concepts which are valid

* Postal address: Laboratoire de Physique Théorique, Faculté des Sciences, Parc Valrose, 06 - Nice (France).

1 Based on part of the author's "Thèse de Doctorat" [1]. 
in both cases. Indeed there appear to be widespread misunderstandings on these points in the current literature and textbooks, particularly in what concerns the notion of intrinsic spin and magnetic moment for elementary systems (particles).

We are here interested in a description of "nonrelativistic elementary particles" and their properties. The frame of our investigation is furnished by the classical analysis of invariance in QM, due to WIGNER [2], and according to which the state space of any quantum mechanical system is a representation space for the relevant kinematical group: the Poincaré group for RQM, the Galilei group for NQM. It is natural to call a system "elementary" (we will also call such a system a "particle"), if the corresponding representation is irreducible. We will not discuss here the shortcomings of such a concept of "elementarity"; instead, we adopt it as a definition, which we strengthen by requiring that the considered system has no additional structure besides the one associated with the corresponding irreducible representation. The purpose of this last proviso is to exclude from our considerations such systems as nuclei, atoms or molecules in stable states which, though being described by an irreducible representation of the kinematical group, possess a complicated internal structure, not accounted for by this representation.

The purely kinematical properties of an isolated quantum mechanical system, i.e. its behaviour under translations, rotations, uniform motions, are completely described by the associated representation of the kinematical group. At this stage, the notion of wave equation for particles is to be considered as a particular method, in general not the most convenient one, of specifying the representation corresponding to the particle: the invariance of the wave equation under the group operation means that its solutions span a representation space for this group. It has become increasingly clear that, in kinematical calculations, it is much easier to deal directly with the representation of the group in a convenient form, rather than with the wave equation and its solutions.

But suppose we now wish to study how the particle behaves in external fields, for instance in order to know its intrinsic electromagnetic properties. Then we cannot use the representation of the kinematical group which only describes the free particle. This is where the notion of wave equations recovers all of its usefulness. Indeed, wave equations constitute the only tool we know of, which enables us to describe interactions in ordinary (first quantized) QM, via the trick of gauge invariance $^{2}$ (of the second kind). Let us emphasize here that, throughout this paper, we will only consider interactions of particles with external (classical) fields, and not interactions between particles.

${ }^{2}$ This is the place to recall that, in NQM, JAUCH [3] has shown the existence of a relation between Galilean invariance and gauge invariance. 
Let us now precisely state our program: we want to establish wave equations for nonrelativistic particles, derive from these wave equations the properties (essentially the electromagnetic ones) of the particles they describe, and compare the whole theory and its results to the corresponding relativistic ones.

\section{Galilei Group Representations and Nonrelativistic Particles}

According to the point of view advocated in the Introduction, the characterization of nonrelativistic particles is furnished by the theory of unitary irreducible representations of the Galilei group. Let us briefly review the results of this theory. The physical representations of the Galilei group are nontrivial projective (ray) representations [4], the true (vector) representations being devoid of physical content ${ }^{3}$ because they do not permit the existence of any sensible notion of localizability [5]. A physical (unitary irreducible) representation of the Galilei group (strictly speaking: an equivalence class of such representations) is characterized by two parameters: a real positive number $m$ and an integer or half-integer positive number $s[6]$, [7]. The representation space (we now single out a particular element in the equivalence class) can be conveniently chosen as the tensor product of a $(2 s+1)$-dimensional vector space and the space $L^{2}\left(\mathbb{R}^{3}\right)$ of square integrable functions on the three-dimensional euclidean space. Let $\Psi_{\alpha}(\mathbf{p}) \quad\left[\mathbf{p} \in \mathbb{R}^{3}\right.$; $\alpha=-s, \ldots,+s]$ be any element of this representation space. Let $(b, \mathbf{a}, \mathbf{v}, R)$ be the generic element of the Galilei group with $b$ a time translation, a a space translation, $\mathbf{v}$ a pure Galilei transformation and $R$ a rotation. The considered representation of the Galilei group is explicitly given by:

$[U(b, \mathbf{a}, \mathbf{v}, R) \Psi]_{\alpha}(\mathbf{p})$

$$
=\exp (i E b-i \mathbf{p} \cdot \mathbf{a}) \times \sum_{\beta=-s}^{+s} D_{\alpha \beta}^{s}(R) \Psi_{\beta}\left(R^{-1}(\mathbf{p}-m \mathbf{v})\right)
$$

where $E=\frac{\mathbf{p}^{2}}{2 m}$ and $D^{s}$ is the $(2 s+1)$-dimensional representation of the rotation group. This representation is unitary for the scalar product corresponding to the following definition of the norm:

$$
\|\Psi\|^{2}=\sum_{\alpha=s}^{+s} \int d^{3} p\left|\Psi_{\alpha}(\mathbf{p})\right|^{2}
$$

The physical interpretation is straightforward: $\Psi_{\alpha}(\mathbf{p})$ is the wave equation in momentum space of a particle with mass $m$ and spin $s$. The kinematical properties of this particle are completely described by the representation (1).

3 Except for a certain class of such representations which have been shown to describe "massless nonrelativistic particles" [7]. 
Let us mention the peculiar role played by the mass. Corresponding to its appearance as characterizing the projective representations of the Galilei group, the mass operator generates a superselection rule [4] (Bargmann's superselection rule), which has important consequences [7], [8]. But we will not be concerned here with this aspect of galilean invariance.

The main point we wish to emphasize is the natural appearance of the concept of spin. It comes out here much in the same way as in RQM [2]. Accordingly, spin is not due to "relativistic effects", is not a "consequence of Dirac equation". Even in NQM, spin has not to be considered as an "extraneous hypothesis", an "independent addition", but on the contrary, follows at once from the first principles.

Having thus shown that spin is not a characteristic feature of RQM, one may well wonder what are these features? In order to answer this question, we clearly have to construct a theory of nonrelativistic particles and their properties which could be compared to the relativistic theory. We will therefore attempt to derive wave equations for nonrelativistic particles of any spin. We open this program by investigating the Galilean invariance of the standard nonrelativistic equation, the Schrödinger equation, in order to understand its connection with the theory of representations of the Galilei group.

\section{Galilean Invariance of the Schrödinger Equation}

The motivation for the present considerations on the Schrödinger equation is twofold: first, its Galilean invariance is overlooked in most textbooks (at best, one finds it relegated to the minor rank of an exercise), though it is no less interesting and much easier to study than the thoroughly studied Lorentz invariance of the Dirac equation; secondly, we may hope to get some hint about the derivation of other nonrelativistic wave equations.

The Schrödinger equation for a particle with mass $m$ writes (we put $\hbar=1)$ :

$$
i \frac{\partial \Phi}{\partial t}=-\frac{1}{2 m} \Delta_{x} \Phi
$$

The wave functions $\Phi$ are complex valued functions of the time $t$ and the spatial coordinates $\mathbf{x}$, with respect to which they are square integrable:

$$
\|\Phi\|^{2}=\int|\Phi(\mathbf{x}, t)|^{2} d^{3} x<+\infty .
$$

By virtue of the equation (3), the norm $\|\Phi\|$ is actually time independent.

Let the particle be subjected to a general Galilei transformation $(b, \mathbf{a}, \mathbf{v}, R)$. Galilean invariance demands the behaviour of the particle to be governed by the same equation (3) after the transformation. Moreover, if such a transformation is not to modify the physical properties 
of the particle, the localization probability density of the particle at a given point before the transformation must be equal to the localization probability density of the particle at the transformed point after the transformation. In other terms:

$$
\left|\Phi^{\prime}\left(\mathrm{x}^{\prime}, t^{\prime}\right)\right|^{2}=|\Phi(\mathrm{x}, t)|^{2}
$$

where $\Phi$ and $\Phi^{\prime}$ are the wave functions of the particle respectively before and after the transformation and the points $(\mathbf{x}, t)$ and $\left(\mathbf{x}^{\prime}, t^{\prime}\right)$ are related by the considered galilean transformation:

$$
\left\{\begin{array}{l}
\mathbf{x}^{\prime}=R \mathbf{x}+\mathbf{v} t+\mathbf{a} \\
t^{\prime}=t+b
\end{array}\right.
$$

The two wave functions thus differ by a phase factor:

$$
\Phi^{\prime}\left(\mathbf{x}^{\prime}, t^{\prime}\right)=e^{i f(\mathbf{x}, t)} \Phi(\mathbf{x}, t)
$$

and it must be possible to find a phase function $f$ such that $\Phi^{\prime}$ obeys the Schrödinger equation (3) as soon as $\Phi$ does. This is indeed the case. One finds after a simple calculation:

$$
f(\mathbf{x}, t)=\frac{1}{2} m \mathbf{v}^{2} t+m \mathbf{v} \cdot R \mathbf{x}+C .
$$

where the constant $C$ could depend on the considered Galilei transformation.

We have thus shown that the Schrödinger equation is Galilean invariant and, moreover, we have obtained the explicit transformation law of the wave functions under a Galilean transformation. The correspondence (7) $\Phi \rightarrow \Phi^{\prime}$ is a unitary ray representation of the Galilei group. Since the Schrödinger equation describes spinless particles with mass $m$, this representation is necessarily equivalent to the corresponding momentum space representation (1) (with $s=0$ ). This equivalence is readily demonstrated by means of a simple Fourier transform. Indeed, there is a one-to-one correspondence between the space of square integrable functions in momentum space and the space of square integrable solutions of the Schrödinger equation (3) (completed with respect to the scalar product associated with the norm (4)) according to:

$$
\Phi(\mathbf{x}, t)=\int e^{-i E t+i \mathbf{p} \cdot \mathbf{x}} \Psi(\mathbf{p}) d^{3} p
$$

where $E=\frac{\mathbf{p}^{2}}{2 m}$. The transformation law (1) then agrees with (7), (8) for $C=0$.

It is now clear that the kinematical descriptions of a free nonrelativistic spinless particle by the associated irreducible representation of the Galilei group, or by the Schrödinger equation, are completely equivalent. As pointed out in the Introduction, if we prefer the group 
representation for describing the free particle, the unchallenged merit of the Schrödinger equation is to allow for the description of the particle interacting with an external field, via the gauge invariance trick of replacing:

$$
\left\{\begin{array}{rrr}
i \frac{\partial}{\partial t} & \text { by } & i \frac{\partial}{\partial t}-\mathscr{V}(\mathbf{x}, t) \\
-i \boldsymbol{\nabla}_{x} & \text { by } & -i \boldsymbol{\nabla}_{x}-\mathscr{A}(\mathbf{x}, t)
\end{array}\right.
$$

$(\mathscr{V}, \mathscr{A})$ being the 4-potential of the field. Remark that one should question the internal consistency of such a scheme where one introduces electromagnetic fields (obeying typically relativistic equations) in nonrelativistic situations. We shall discuss this problem in VI.

\section{A Nonrelativistic Dirac Equation}

\section{a) Wave Equations for Nonzero Spin?}

We will now try to obtain wave equations for nonrelativistic particles of any spin, taking the opposite way to the one we just followed, i.e. we start from the irreducible representation of the Galilei group to reach a related wave equation which, though equivalent to the considered representation in the free particle case, can be used in the presence of interaction.

We have first to go from the momentum space representation (1) to some configuration space representation. Indeed, only in configuration space does the idea of wave equation as a partial derivative equation make sense; moreover, when interaction is present, it is only in configuration space that such notions as forces, potentials, are physically defined. Using the approach of NEwTON and WIGNER to localizability problems [9], it can be shown that a particle described by a representation (1) is localizable for any value of the spin, if it has a nonzero mass. The position operator corresponding to this notion of localizability is simply given, in momentum space, by:

$$
\mathbf{X}=i \boldsymbol{\nabla}_{p}
$$

this being valid independently of the spin of the particle. That means that we obtain an a priori acceptable configuration space representation equivalent to (1), by performing a Fourier transformation in a way completely analogous to the spinless case. It suffices to use eq. (9) for the $(2 s+1)$-component momentum space wave function, thus getting $(2 s+1)$-component wave functions $\Phi_{\alpha}(\mathbf{x}, t)$ defined in configuration space. The Galilei group representation now takes the form:

$$
\Phi_{\alpha}^{\prime}\left(\mathbf{x}^{\prime}, t^{\prime}\right)=e^{i f(\mathbf{x}, t)} \sum_{\beta} D_{\alpha \beta}^{s}(R) \Phi_{\beta}(\mathbf{x}, t)
$$

with the same phase function $f(8)$ as in the spinless case (7). This representation is unitary for the scalar product corresponding to the definition 
of the norm:

$$
\|\Phi\|^{2}=\sum_{\alpha=-s}^{+s} \int\left|\Phi_{\alpha}(\mathrm{x}, t)\right|^{2} d^{3} x .
$$

This expression is time independent, due to (12). The law (12) is a satisfying transformation law in that it is local, the value of the new function $\Phi^{\prime}$ at the transformed point depending only on the value of the function $\Phi$ at the initial point. Things are simpler here than in the relativistic case where the dependence of the little group operators on the momentum $p$ [2] requires a generalized Foldy-Wouthuysen transformation to be made on Wigner's representation before a local configuration space wave-function may be obtained by Fourier transformation.

We now search for an invariant wave equation associated with the transformation law (12). What we mean is: does there exist a partial derivative equation such that it is automatically obeyed by $\Phi^{\prime}$, if it is obeyed by $\Phi, \Phi$ and $\Phi^{\prime}$ being related by (12)? In other words, we are looking for a Galilei invariant operator formed from $\frac{\partial}{\partial t}, \boldsymbol{\nabla}_{x}$ and the spin operators in the $(2 s+1)$-dimensional space. A tedious but straightforward calculation yields that the only solutions to this problem are the functions of the Schrödinger operator:

$$
S \stackrel{\text { def }}{=} i \frac{\partial}{\partial t}+\frac{1}{2 m} \Delta=E-\frac{1}{2 m} \mathbf{p}^{2}
$$

The spin operators do not appear if we pose the problem in this way. It should be remarked that the situation is the same in the case of the Poincaré group. Wave equations which couple the different spin states are possible only if one allows for wave equations with more than $(2 s+1)$ components.

\section{b) Linearization of the Schrödinger Equation}

We shall now derive such a wave equation, which will turn out to describe spin $1 / 2$ particles, using the heuristic idea that Dirac applied so successfully in RQM [10]; we require the equation to be of first-order in all the space-time derivatives. Thus, we search a wave equation in the form:

$$
\theta \Phi \stackrel{\text { de } \mathrm{i}}{=}(A E+\mathbf{B} \cdot \mathbf{p}+C) \Phi=0,
$$

$A, \mathbf{B}, C$ being linear operators to be determined, operating in a vector space whose dimension we still ignore but which we will assume to be finite.

For the solutions of (15) to obey the Schrödinger equation:

$$
S \Phi=0
$$

there must exist some operator $\theta^{\prime}=A^{\prime} E+\mathbf{B}^{\prime} \cdot \mathbf{p}+C^{\prime}$, such that multiplying (15) by $\theta^{\prime}$ we recover the Schrödinger equation. In other 
words we must have

$$
\theta^{\prime} \theta=2 m S
$$

the arbitrary numerical coefficient $2 \mathrm{~m}$ providing a convenient normalization. By identifying the various monomials in $E$ and $\mathbf{p}$, we obtain the following set of conditions:

$$
\left\{\begin{array} { l } 
{ A ^ { \prime } A = 0 } \\
{ A ^ { \prime } C + C ^ { \prime } A = 2 m } \\
{ C ^ { \prime } C = 0 }
\end{array} \left\{\begin{array}{l}
A^{\prime} B_{i}+B_{i}^{\prime} A=0 \\
B_{i}^{\prime} B_{\jmath}+B_{j}^{\prime} B_{i}=-2 \delta_{i j} \quad(i, j=1,2,3) \\
C^{\prime} B_{i}+B_{i}^{\prime} C=0 .
\end{array}\right.\right.
$$

Defining the new operators:

$$
\begin{array}{ll}
B_{4}=i\left(A+\frac{1}{2 m} C\right) & B_{4}^{\prime}=i\left(A^{\prime}+\frac{1}{2 m} C^{\prime}\right) \\
B_{5}=A-\frac{1}{2 m} C & B_{5}^{\prime}=A^{\prime}-\frac{1}{2 m} C^{\prime}
\end{array}
$$

the conditions (18) can be rewritten as:

$$
B_{\mu}^{\prime} B_{\nu}+B_{\nu}^{\prime} B_{\mu}=-2 \delta_{\mu \nu} \cdot(\mu, v=1 \text { to } 5)
$$

All the representations of such an algebra can be obtained from those of a Clifford algebra with dimension 4:

$$
\gamma_{\alpha} \gamma_{\beta}+\gamma_{\beta} \gamma_{\alpha}=2 \delta_{\alpha \beta} \quad(\alpha, \beta=1 \text { to } 4)
$$

according to the correspondence:

$$
\begin{array}{ll}
B_{\alpha}=B \gamma_{\alpha} & B_{\alpha}^{\prime}=-\gamma_{\alpha} \beta^{-1} \quad(\alpha=1 \text { to } 4) \\
B_{5}=-i \beta & B_{5}^{\prime}=-i \beta^{-1}
\end{array}
$$

where $\beta$ is an arbitrary nonsingular matrix. Since we are only interested in the irreducible representations, we may use the standard results for the Dirac algebra (21). All the irreducible representations then have dimension 4 and are equivalent. We choose the following realization, standard for the Dirac equation, where the $\sigma_{\imath}$ 's are the Pauli matrices:

So that, after (19):

$$
\begin{aligned}
& B_{i}=\left(\begin{array}{ll}
\sigma_{i} & 0 \\
0 & \sigma_{i}
\end{array}\right) \quad(i=1,2,3) \\
& B_{4}=\left(\begin{array}{ll}
0 & 1 \\
1 & 0
\end{array}\right), \quad B_{5}=\left(\begin{array}{rr}
0 & -i \\
i & 0
\end{array}\right)
\end{aligned}
$$

$$
A=\left(\begin{array}{ll}
0 & 0 \\
1 & 0
\end{array}\right), \quad C==\left(\begin{array}{ll}
0 & 2 m \\
0 & 0
\end{array}\right)
$$

The wave function $\Phi$ is thus a 4 -component object, which we write as $\Phi=\left(\begin{array}{l}\varphi \\ \chi\end{array}\right), \varphi$ and $\chi$ each being a 2 -component function. Our wave equation finally reads:

$$
\left\{\begin{array}{l}
E \varphi+(\boldsymbol{\sigma} \cdot \mathbf{p}) \chi=0 \\
(\boldsymbol{\sigma} \cdot \mathbf{p}) \varphi+2 m \chi=0
\end{array}\right.
$$


By direct substitution, one finds that both $\varphi$ and $\chi$ satisfy the Schrödinger equation. Since the $A$ matrix in (15) is singular (see (24)), the wave equation (25) considered as an eigenvalue equation for the energy has only two identical eigenvalues, $E=\frac{\mathbf{p}^{2}}{2 m}$ precisely. The corresponding eigenvectors are of the form $\Phi=\left(\begin{array}{c}\varphi \\ -\frac{1}{2 m}(\boldsymbol{\sigma} \cdot \mathbf{p}) \varphi\end{array}\right)$. In that sense, these 4-components might seem to contain an useless redundancy. However, this is not the case, as will be seen when we introduce the effect of an external electromagnetic field by the usual substitution (10).

\section{c) Galilean Invariance and the Interpretation of the Wave Equation}

Before definitively accepting the wave equation and using it, it is necessary to prove its galilean invariance in order to justify our previous heuristic derivation. Calling $\Phi^{\prime}=\left(\begin{array}{l}\varphi^{\prime} \\ \chi^{\prime}\end{array}\right)$ the wave function transformed from $\Phi=\left(\begin{array}{l}\varphi \\ \chi\end{array}\right)$ by the general galilean transformation $(b, \mathbf{a}, \mathbf{v}, R)$ which takes $(\mathbf{x}, t)$ into $\left(\mathbf{x}^{\prime}, t^{\prime}\right)(6)$, one finds that the wave equation (25) is invariant under the substitution:

$$
\left(\begin{array}{l}
\varphi^{\prime}\left(\mathbf{x}^{\prime}, t^{\prime}\right) \\
\chi^{\prime}\left(\mathbf{x}^{\prime}, t^{\prime}\right)
\end{array}\right)=e^{\mathrm{if}(\mathbf{x}, t)}\left(\begin{array}{cc}
D^{1 / 2}(R) & 0 \\
-\frac{\boldsymbol{\sigma} \cdot \mathbf{v}}{2} D^{1 / 2}(R) & D^{1 / 2}(R)
\end{array}\right)\left(\begin{array}{l}
\varphi(\mathbf{x}, t) \\
\chi(\mathbf{x}, t)
\end{array}\right)
$$

where the phase function $f$ is the same as in the Schrödinger case (8), and $D^{1 / 2}$ is the two-dimensional ray representation of the rotation group. The $\varphi$ component transforms very simply, without mixing with the $\chi$ component. In its transformation law

$$
\varphi^{\prime}\left(\mathbf{x}^{\prime}, t^{\prime}\right)=e^{\mathrm{if}(\mathbf{x}, t)} D^{1 / 2}(R) \varphi(\mathbf{x}, t)
$$

we recognize the particular case $s=1 / 2$ of the general expression (12) of the Galilei group irreducible representations. Since the function $\varphi$ suffices to describe all the physical properties of a free system, the wave equation (25) describes a nonrelativistic particle with mass $m$ and spin $1 / 2$.

Returning to the transformation properties (26) of the complete wave function, we find out a remarkable fact: the $4 \times 4$ matrices

$$
\Delta^{1 / 2}(\mathbf{v}, R) \stackrel{\text { def }}{=}\left(\begin{array}{cc}
D^{1 / 2}(R) & 0 \\
-\frac{\boldsymbol{\sigma} \cdot \mathbf{v}}{2} D^{1 / 2}(R) & D^{1 / 2}(R)
\end{array}\right)
$$

make up a faithful representation of the "homogeneous Galilei group", i.e. obey the multiplication law:

$$
\Delta^{1 / 2}\left(\mathbf{v}^{\prime}, R^{\prime}\right) \Delta^{1 / 2}(\mathbf{v}, R)=\Delta^{1 / 2}\left(\mathbf{v}^{\prime}+R^{\prime} \mathbf{v}, R^{\prime} R\right)
$$


which is readily verified, using the standard relation:

$$
D^{1 / 2}\left(R^{-1}\right) \boldsymbol{\sigma} D^{1 / 2}(R)=R \boldsymbol{\sigma}
$$

The wave function $\Phi=\left(\begin{array}{l}\varphi \\ \chi\end{array}\right)$ thus appears to take its values in a finite dimensional representation space of the homogeneous Galilei group.

\section{d) The Particle in an External Field and its Magnetic Moment}

It is time to draw more explicit conclusions from our wave equation (25). Let us consider the effect of an electromagnetic field on the particle. Here also, it might be questioned if the introduction of such a field, typically relativistic since obeying Maxwell equations, is not to destroy the internal consistency of a Galilean theory; we will show (VI) that, at least in the case of static fields, this is not so. Using gauge invariance of the second kind to introduce the fields $(\mathscr{V}, \mathscr{A})$ (via the replacement $(10)$ ), the wave equation for a nonrelativistic particle with mass $m$, spin $1 / 2$ and charge $q$ reads :

$$
\left\{\begin{array}{l}
(E-q \mathscr{V}) \varphi+\boldsymbol{\sigma} \cdot(\mathbf{p}-q \mathscr{A}) \chi=0 \\
\boldsymbol{\sigma} \cdot(\mathbf{p}-q \mathscr{A}) \varphi+2 m \chi=0
\end{array}\right.
$$

We eliminate the auxiliary components $\chi$, thereby obtaining the following physical wave equation for $\varphi$ :

$$
\left[E-q \mathscr{V}-\frac{1}{2 m}(\mathbf{p}-q \mathscr{A})^{2}-\frac{q}{2 m} \boldsymbol{\sigma} \cdot \mathscr{H}\right] \varphi=0
$$

where $\mathscr{H}=\operatorname{rot} \mathscr{A}$ is the magnetic field. Obviously, what makes this equation interesting is the last term which proves the existence of an intrinsic magnetic moment for the particle, given by:

$$
\boldsymbol{\mu}=\frac{q}{2 m} \boldsymbol{\sigma}
$$

Since the spin of the particle is $\mathrm{S}=\frac{1}{2} \boldsymbol{\sigma}$, its gyromagnetic ratio is $\gamma=\frac{q}{m}$, i.e. twice the value of the orbital ratio, so that the spin Landé factor reads $g_{s}=2$.

A complete nonrelativistic theory predicts the correct ${ }^{4}$ value for the intrinsic magnetic moment of a spin $1 / 2$ particle.

This phenomenon, which caused so much trouble to physicists in the twenties and which Dirac first explained by means of his equation, is thus shown not to be a specific consequence of relativistic invariance. The "extraordinary" value of the spin gyromagnetic ratio, like the very

${ }^{4}$ As emphasized in the Introduction, the particles here considered are supposed to be completely described by their wave equation, i.e. there are no strong interactions, neither radiative corrections to modify the derived value of the magnetic moment. 
existence of the spin, is nothing of a "relativistic effect" as is plainly or tacitly stated in many textbooks ${ }^{5}$.

Of course, our final equation (32) is nothing but the usual Pauli equation [11]. The novelty of our approach lies in the fact that:

i) the spin degrees of freedom do not have to be introduced "ad hoc", but are contained in the theory from its starting point, equation (25).

ii) the correct value of the magnetic moment results from the theory and does not need to be taken out from experimental results. Our derivation, moreover, automatically implies the Galilean invariance of the Pauli equation.

It must be added that eq. (32) shows no evidence for spin-orbit interaction or the Darwin term. These are truly relativistic effects, only predicted by the Dirac equation and whose practical importance is a sufficiently striking success of this equation.

GALINDO and SANCHEZ DEL RIO (12], had already announced such a result on the nonrelativistic nature of the extraordinary spin magnetic moment. However, we do not think their derivation to be quite satisfying: they linearize the Schrödinger equation in such a way as to introduce the square root of the energy operator. And worse, their equation is not invariant under the whole Galilei group but only under its subgroup which they call "static" and which does not include the pure Galilei transformations. Let us also mention that EBERLEIN [13] obtained the correct magnetic moment in the Pauli equation from very different considerations, of a formal mathematical nature.

\section{e) Probability Density and Current}

In order to apply the wave equation (25) to the analysis of physical problems, we have to know some sesquilinear form in the wave function $\Phi$, which can be used as a probability density. This form may be determined by the condition that the associated norm of $\Phi$ be time-independent by virtue of the wave equation. Let us write the wave equation in the form:

$$
\left\{\begin{array}{l}
i \frac{\partial \varphi}{\partial t}-i \boldsymbol{\sigma} \cdot \boldsymbol{\nabla} \chi=0 \\
-i \boldsymbol{\sigma} \cdot \boldsymbol{\nabla} \varphi+2 m \chi=0 .
\end{array}\right.
$$

The norm of $\Phi$ must be defined by:

$$
\|\Phi\|^{2}=\int \varphi^{+} \varphi d^{3} x
$$

${ }^{5}$ It might be that these unfounded assertions result in part from the use of a unit system in which the Bohr magneton writes $\mu_{B}=\frac{e}{2 m c}$ (instead of $\frac{e}{2 m}$ here), the presence of the constant $c$ giving a "relativistic look" to such a formula. In this connection we will see later that dimensional arguments, when carefully handled, give interesting results (IV.c). 
in order to be time-independent according to (34). We then define the probability density:

$$
\varrho=\varphi^{+} \varphi \text {. }
$$

This sesquilinear form, in spite of its appearance, is nondegenerate; indeed, $\varrho=0$ demands $\varphi=0$, which, in turn, implies $\chi=0$ after (34), so that finally $\Phi=0$; from the galilean transformation law (27), it is seen that the probability density $\varrho$ is a galilean scalar, in accordance with its physical meaning, as in Schrödinger case.

To the conserved norm (35), there corresponds a continuity equation

$$
\frac{\partial \varrho}{\partial t}+\operatorname{div} \mathbf{j}=0
$$

where $\mathbf{j}$ is a probability density current. After the wave equation (34) and its adjoint, we may write:

$$
\begin{aligned}
\frac{\partial \varrho}{\partial t} & =\varphi^{+} \frac{\partial \varphi}{\partial t}+\frac{\partial \varphi^{+}}{\partial t} \varphi \\
& =\varphi^{+}(\boldsymbol{\sigma} \cdot \boldsymbol{\nabla} \chi)+\left(\boldsymbol{V} \chi^{+} \cdot \boldsymbol{\sigma}\right) \varphi \\
& =\operatorname{div}\left(\varphi^{+} \boldsymbol{\sigma} \chi+\chi^{+} \boldsymbol{\sigma} \varphi\right) .
\end{aligned}
$$

The simplest choice for the current, that is

$$
\mathbf{j}=-\left(\varphi^{+} \boldsymbol{\sigma} \chi+\chi^{+} \boldsymbol{\sigma} \varphi\right)
$$

is the only one compatible with the minimal electromagnetic coupling discussed above. Indeed, a more transparent expression for this current $\mathbf{j}$ is obtained by eliminating the auxiliary components $\chi$ with the help of equation (34). Expressed in term of $\varphi$ only, we obtain, after manipulating some Pauli matrices:

$$
\mathbf{j}=\frac{1}{2 m i}\left[\varphi^{+}(\boldsymbol{\nabla} \varphi)-\left(\boldsymbol{\nabla} \varphi^{+}\right) \varphi\right]+\frac{1}{2 m} \boldsymbol{\nabla} \times\left(\varphi^{+} \boldsymbol{\sigma} \varphi\right) .
$$

The first term on the right-hand side is completely analogous to the usual expression for the probability density current of the Schrödinger equation. The second term is a spin current, which gives rise to the correct value (33) of the spin magnetic moment when the current (40) is inserted in the standard electromagnetic interaction Lagrangian:

$$
L_{\mathrm{I}}=q \int(\varrho \mathscr{V}-\mathbf{j} \cdot \mathscr{A}) d^{3} x .
$$

In fact, the spin part of this Lagrangian may be cast in the form:

$$
\begin{aligned}
L_{\mathrm{I} s} & =-\frac{q}{2 m} \int\left[\boldsymbol{\nabla} \times\left(\varphi^{+} \boldsymbol{\sigma} \varphi\right)\right] \cdot \mathscr{A} d^{3} x \\
& =\frac{q}{2 m} \int\left(\varphi^{+} \boldsymbol{\sigma} \varphi\right) \cdot \mathscr{H} d^{3} x .
\end{aligned}
$$

One recognizes the existence of an intrinsic magnetic moment $\boldsymbol{\mu}=\frac{q}{2 m} \boldsymbol{\sigma}$. 21 Commun. math. Phys., Vol. 6 


\section{f) Connection with the Dirac Equation}

Let us write the Dirac equation in the following way:

$$
\left\{\begin{array}{l}
(\mathscr{E}-m) \varphi+(\boldsymbol{\sigma} \cdot \boldsymbol{p}) \chi=0 \\
(\boldsymbol{\sigma} \cdot \boldsymbol{p}) \varphi+(\mathscr{E}+m) \boldsymbol{\chi}=0
\end{array}\right.
$$

where $\mathscr{E}$ is the total energy (mass + kinetic) and $\varphi, \chi$ two 2 -component spinors (usually termed big and small component respectively of the Dirac wave function). In the nonrelativistic limit where $\mathscr{E}=m+E$, $E \ll m$, it is natural to write equation (43) in the approximate form (25), thus recovering in almost a trivial way our wave equation, which appears very simply as the nonrelativistic limit of the Dirac equation. As a matter of fact, some authors have written down this equation when studying the Dirac equation and its nonrelativistic limit. However, they usually jump over to the Schrödinger equation which results from this, losing in the process many interesting features. On the contrary, it is most rewarding to pause a while at this stage, in order to exhibit the Galilean invariance of this nonrelativistic equation. While the usual way of identifying the magnetic moment of a Dirac particle consists in introducing the electromagnetic fields in the Dirac equation and then going to the nonrelativistic limit, we have shown that it is sufficient to introduce the electromagnetic fields, after taking the nonrelativistic limit.

\section{Nonrelativistic Wave Equations for any Spin}

\section{a) General Remarks}

In $\mathrm{RQM}$, the relation between irreducible representations of the Poincaré group and wave equations by no means is a simple one. While a particle with spin $s$ is described, in WIGNER's standard form of the irreducible representations, by a $(2 s+1)$-component wave function [2], non-trivial wave equations can only be written for wave functions with a larger number of components ${ }^{6}$. The wave equation then may be viewed as simultaneously expressing constraints on the "redundant" components and equations of motion for the "physical" ones. But the separation is not a covariant one. In fact, it turns out that the usual wave equations are characterized by a representation of the homogeneous Lorentz group according to which the components of the wave function transform. The appearance of the Lorentz group, instead of the rotation group (little group) in WIGNER's theory, is rather mysterious.

The same mystery is present in NQM. Although we do not understand at present its physical meaning, we nevertheless adopt the same guideline as in the relativistic case on our way from the irreducible representation of the invariance group to a wave equation. Specifically,

${ }^{6}$ For a recent comprehensive article on relativistic wave equations, containing numerous references to original papers, see ref. [14]. 
we will require the components of the wave function to transform according to some finite-dimensional representation $\Delta$ of the homogeneous Galilei group:

$$
\Phi^{\prime}\left(\mathbf{x}^{\prime}, t^{\prime}\right)=e^{\text {if }(\mathbf{x}, t)} \Delta(\mathbf{v}, R) \Phi(\mathbf{x}, t)
$$

where $f$ is the standard phase function. It is such a transformation law which we already met in the spin $1 / 2$ case (26). Since any wave equation can be re-expressed as a first order equation, we could look for the more general invariant equation of the form (15). This is the method used by GEL'FAND and YAGLOM in RQM?. It leads to a simultaneous determination of the representation $A$ and the operators $(A, \mathbf{B}, C)$. Unfortunately, the homogeneous Galilei group (isomorphic to the three-dimensional euclidean group) is not semi-simple and its finite dimensional representations are rather complicated to classify [16]. In particular, if faithful, they are necessarily nilpotent and thus cannot be irreducible, but at most undecomposable (such is the case for the representation (28)). It is not easier to construct them by contracting [17] finite-dimensional representation of the Lorentz group, since it does not suffice to consider the well-known irreducible finite-dimensional representations $\mathscr{D}^{j}, j^{j}$; for instance, the undecomposable representation (28) derives by contraction from the reducible Dirac representation $\mathscr{D}^{0,1 / 2} \oplus \mathscr{D}^{1 / 2,0}$ of the Lorentz group.

\section{b) The Bargmann-Wigner Method}

We thus try another approach which will prove more rewarding, using Bargmann-Wigner method [18] in our Galilean situation. Consider the complex valued functions in the coordinates $(\mathbf{x}, t)$ and in $N$ discrete four-valued variables $\lambda_{i}\left(\lambda_{i}=1,2,3,4 ; i=1, \ldots, N\right)$. We require these functions $\Phi\left(\mathbf{x}, t ; \lambda_{1}, \ldots, \lambda_{N}\right)$ to be symmetrical in the $N$ variables $\lambda$, which already reduces the number of independent components from $4^{N}$ to $\frac{(N+3) !}{N ! 3 !}$. For each value of $i=1, \ldots, N$, one defines an operator $\theta_{i}$ (see III. b), which acts trivially on the indices $\lambda$, except the $i^{\text {th }}$ on which it operates according to the definition:

$$
\theta_{i} \stackrel{\text { def }}{=}\left(\begin{array}{cc}
E & \boldsymbol{\sigma} \cdot \mathbf{p} \\
\boldsymbol{\sigma} \cdot \mathbf{p} & 2 m
\end{array}\right)_{\imath} \text {. }
$$

The function $\Phi$ is now required to obey the following set of equations:

$$
\theta_{i} \Phi=0 \quad i=1, \ldots, N
$$

in complete analogy to the relativistic Bargmann-Wigner wave equations. Let us define the norm of the state represented by the wave function $\Phi$ by :

$$
\|\Phi\|^{2}=\sum_{\lambda_{1}=1}^{2} \cdots \sum_{\lambda_{N}=1}^{2} \int\left|\Phi\left(\mathrm{x}, t ; \lambda_{1}, \ldots, \lambda_{N}\right)\right|^{2} d^{3} x
$$

3 Their work is reviewed and the references to the original papers (in Russian) are given in ref. [15], part II, chap. II. 
It is non-degenerate and time-independent by virtue of the wave equations (46) (compare the corresponding discussion in III.e). This definition of the norm endows the space of wave functions with a Hilbert space structure; in this Hilbert space operates an unitary representation of the Galilei group given by:

$$
\Phi^{\prime}\left(\mathbf{x}^{\prime}, t^{\prime}\right)=e^{\mathrm{if}(\mathbf{x}, t)} \delta^{\left[{ }^{\mathrm{y}}\right]}(\mathbf{v}, R) \Phi(\mathbf{x}, t)
$$

where $f$ is the usual phase function $(8),\left(\mathbf{x}^{\prime}, t^{\prime}\right)$ depends on $(\mathbf{x}, t)$ according to (6) and $\delta^{[N]}$ is the completely symmetrized tensor product of $N$ representations $\delta^{[1]} \stackrel{\text { def }}{=} \Delta^{1 / 2}$ (defined by eq. (40)) of the homogeneous Galilei group. The operators $\delta^{[N]}$ of these representations are linear operators acting on the indices $\lambda$ of the wave function. The invariance of the wave equation (46) under the representation (48) of the Galilei group is easily proved, as in the spin $1 / 2$ case (see III.c).

We are left with the problem of the physical interpretation of these wave equations. What kind of particles do they represent? This should be answered by reducing the representation (48) into a sum of irreducible components. But we may proceed more rapidily. As a matter of fact, in this reduction will appear various irreducible representations corresponding to the redundant components of the wave function in which we are not interested, since they do not carry direct information on the particle described by the wave equations (46). Instead, it suffices to consider the independent components of the wave function. Exactly as in the spin $1 / 2$ case, we use the wave equations themselves to eliminate all the components $\Phi(\mathrm{x}, t ; \lambda)$ where one index $\lambda$ at least takes the values $\lambda_{i}=3,4$; by expressing such components in terms of the components $\Phi(\mathrm{x}, t ; \lambda)$ where each index $\lambda$ only takes the values $\lambda_{i}=1,2$, we are left with a priori $2^{N}$ components. But the symmetry of $\Phi$ in the variables $\lambda$ reduces the number of independent components to $(N+1)$ (this is the number of ways of distributing the values 1 and 2 between the $N$ indices $\lambda$ without taking their order into account). Due to the definition of the representation $\delta^{[N]}$, this $(N+1)$-component object $\varphi$ transforms according to the following representation of the Galilei group:

$$
\varphi^{\prime}\left(\mathbf{x}^{\prime}, t^{\prime}\right)=e^{\mathrm{if}(\mathbf{x}, t)} d^{[N]}(\mathbf{v}, R) \varphi(\mathbf{x}, t)
$$

where $d^{[N]}$ is the $(N+1)$-dimensional representation of the Galilei group obtained by restricting the matrices of the representation $\delta^{[N]}$ to the (invariant) subspace defined by the values $\lambda_{i}=1,2$ of the indices $\lambda$. According to the expression (40) of the matrices $\Delta^{1 / 2}$ whose symmetrized tensor product defines $\delta^{[N]}, d^{[N]}$ is but the symmetrized tensor product of $N$ irreducible representations $D^{1 / 2}\left(=d^{[1]}\right)$ of the rotation group, so that

$$
d^{[N]}(\mathbf{v}, R)=D^{N / 2}(R)
$$

where $D^{x / 2}$ is the irreducible $(N+1)$-dimensional representation of the 
rotation group. The independent components of the wave equations thus transform according to:

$$
\varphi^{\prime}\left(\mathbf{x}^{\prime}, t^{\prime}\right)=e^{\mathrm{if}(\mathbf{x}, t)} D^{N / 2}(R) \varphi(\mathbf{x}, t)
$$

in which one recognizes an irreducible representation of the Galilei group: see equation (12). Therefore:

The equations "à la Bargmann-Wigner" (46) describe a nonrelativistic particle with spin $N / 2$ and mass $m$.

(the mass $m$ appears through the phase function $f(8)$ ).

Of course, these wave equations are much more complicated than the representation (51), but they will enable us to study nonrelativistic particles interacting with external fields. However, for that purpose, the equations (46) as they stand, are not yet very suitable, because of the great number of redundant components, so that it is useful to cast them in a somewhat more tractable form. We will illustrate this process and the subsequent use of the resulting equations in the case of a spin 1 particle. A further specialization to the zero mass case will yield the equations of "galilean electromagnetism".

\section{c) Electromagnetic Properties of Elementary Systems}

As already emphasized, the main advantage of wave equations is to permit the study of the electromagnetic interactions of elementary systems $^{8}$. It may be said here that the intrinsic magnetic moment for a particle of spin $s \neq 0$, charge $q$ and mass $m$ is found to be, in RQM, $\mu=\frac{q \hbar}{2 m}$ for all spin values where it has been computed, i.e. any halfodd-integer value [19] and $s=1$ [20], 2 [21]. In NQM, we computed the magnetic moment of a spin $1 / 2$ particle and will soon do the same for a spin 1 particle. We find the same value of the magnetic moment and except to obtain it also for any other value of $s$. It is most disconcerting that this simple result needs rather involved computations. A simple argument would be desirable in both RQM and NQM.

Concerning the higher multipole moments, it is easy to see from elementary dimensional analysis that they must vanish in NQM. A mass $m$ and spins particle is said to possess an electric (resp. magnetic) multipole moment in order $l$, or $2^{l}$-polar moment, if the expression for the energy of the particle in an electrostatic (resp. magnetostatic) field linearly depends on the spatial derivatives of order $l$ of the electric (resp. magnetic) potentials. In other words, there exists interaction

8 We recall that, throughout this paper, by "elementary system", we mean a system strictly obeying the ordinary wave equations, i.e. we do not consider radiative corrections neither, a fortiori, strong interaction effects. 
terms such as:

$$
\begin{gathered}
\varepsilon^{(l)} D^{l} \mathscr{V} \\
\mu^{(l)} D^{l} \mathscr{A}
\end{gathered}
$$

where $(\mathscr{V}, \mathscr{A})$ are the electromagnetic potentials and $D^{l}$ some differential operator of order $l$ in the spatial coordinates. The coefficients $\varepsilon^{(l)}$ and $\mu^{(l)}$, suitably normalized, give the value of the electric and magnetic multipole moments of order $l$. Recall that a spin $s$ particle cannot possess multipole moments beyond the order $2 s$, and that elementary considerations about parity (when it is conserved, which we admit) show that the electric (resp. magnetic) multipole moments of odd (resp. even) order identically vanish.

We may derive dimensional equations for the multipole moments by recognizing the quantities (52) to have the dimensions of an energy. Let us choose a unit system with fundamental quantities: length (dimension $L)$, mass $(M)$, time $(T)$ and charge $(Q)$. We obtain immediately:

$$
\begin{aligned}
{\left[\varepsilon^{(l)}\right] } & =Q L^{l} \\
{\left[\mu^{(k)}\right] } & =Q L^{k+1} T^{-1}
\end{aligned}
$$

In a relativistic theory of an elementary system, the only quantities which we may use are the mass $m$ and charge $q$ of the particle along with the universal constants $\hbar$ (Planck's constant) and $c$ (light velocity). These define unambiguously a certain unit system, so that one has necessarily:

$$
\begin{aligned}
\varepsilon^{(l)} & =\alpha_{l} q\left(\frac{\hbar}{m c}\right)^{l} \\
\mu^{(k)} & =\beta_{k} q\left(\frac{\hbar}{m c}\right)^{k} c
\end{aligned}
$$

where $\alpha_{l}, \beta_{k}$ are real numbers.

Let us now remark that in a nonrelativistic theory, the velocity of light is irrelevant and does not enter the equations. As a result, in such a theory, only the quantities (54) which do not depend on $c$, may be obtained. The only possibility thus left to a galilean particle is to possess electromagnetic moments:

$$
\left\{\begin{array}{l}
\varepsilon^{(0)}=\alpha_{0} q \\
\mu^{(1)}=\beta_{1} q \frac{\hbar}{m}
\end{array}\right.
$$

(of course $\alpha_{0}=1$ by definition and we mentioned that $\beta_{1}=\frac{1}{2}$ ).

A nonrelativistic particle cannot possess intrinsic electromagnetic properties besides an electric charge and a magnetic dipole moment.

So that the quadrupole electric moments, etc., for elementary systems are specifically relativistic effects. 
Such a result obviously is wrong if the particle is not "elementary", i.e. if it possesses an internal structure characterized by some supplementary parameters, binding energy, mean radius, etc. Such is the case for the deuteron for instance, so that its nonvanishing quadrupole moment does not contradict our result.

\section{Galilean Particles with Spin 1 and Nonzero Mass}

a) The Representation of the Homogeneous Galilei Group

We shall first derive the representation of the Galilei group corresponding to the transformation properties of the wave function describing a spin 1 galilean particle. The equations (46) "à la BargmannWigner" with $N=2$ constitute our starting point. The representation which we are looking for is given by the general formula (48); it remains to obtain a somewhat more explicit characterization of the representation $\delta^{[2]}$ of the homogeneous Galilei group. This is the symmetrized tensor product of two representations $\Delta^{1 / 2}$. Let $\left(\begin{array}{l}\varphi \\ \chi\end{array}\right)$ denote a standard basis in the representation space of $\Delta^{1 / 2}$ (see III.c). We construct a basis in the representation space of $\delta^{[2]}$ by taking a complete set of independent linear combinations of symmetrical tensor products of two such standard basis $\left(\begin{array}{l}\varphi_{1} \\ \chi_{1}\end{array}\right)$ and $\left(\begin{array}{l}\varphi_{2} \\ \chi_{2}\end{array}\right)$. Defining "adjoint" basis $(\bar{\varphi} \bar{\chi})$ according to:

$$
\bar{\varphi}=\varphi^{T} \sigma_{2} \quad \bar{\chi}=\chi^{T} \sigma_{2}
$$

where $\left(\varphi^{T} \chi^{T}\right)$ is the transpose of $\left(\begin{array}{l}\varphi \\ \chi\end{array}\right)$, we choose the following basis in the ten-dimensional representation space of $\delta^{[2]}$ :

$$
\left\{\begin{array}{l}
\mathbf{U}=\bar{\varphi}_{1} \boldsymbol{\sigma} \varphi_{2} \\
W=\bar{\chi}_{1} \varphi_{2}-\bar{\varphi}_{1} \chi_{2} \\
\mathbf{L}=-m \bar{\chi}_{1} \boldsymbol{\sigma} \chi_{2} \\
\mathbf{M}=i m\left(\bar{\varphi}_{1} \boldsymbol{\sigma} \chi_{2}+\bar{\chi}_{1} \boldsymbol{\sigma} \varphi_{2}\right) .
\end{array}\right.
$$

We now use the explicit form (28) of the representation $\delta^{[1]}=\Delta^{1 / 2}$ of the homogeneous Galilei group under which $\left(\begin{array}{l}\varphi_{1} \\ \chi_{1}\end{array}\right)$ and $\left(\begin{array}{l}\varphi_{2} \\ \chi_{2}\end{array}\right)$ transform, to obtain the transformation properties of the wave-function defined in (57). Using the identity (30), we find:

$$
\left\{\begin{array}{l}
\mathbf{U}^{\prime}=R \mathbf{U} \\
W^{\prime}=W+\mathbf{v} \cdot R \mathbf{U} \\
\mathbf{L}^{\prime}=R \mathbf{L}+\mathbf{v} \times R \mathbf{M}+m\left[\mathbf{v} W+\mathbf{v}(\mathbf{v} \cdot R \mathbf{U})-\frac{1}{2}\left(\mathbf{v}^{2}\right) R \mathbf{U}\right] \\
\mathbf{M}^{\prime}=R \mathbf{M}+m[\mathbf{v} \times R \mathbf{U}] .
\end{array}\right.
$$


It can be checked by a direct and instructive computation that this is a faithful ten-dimensional representation of the homogeneous Galilei group. This representation is reducible but undecomposable (not completely reducible), although it may be obtained by contraction [17] from the completely reducible representation $\mathscr{D}^{1 / 2,1 / 2} \oplus \mathscr{D}^{0,1 / 2} \oplus \mathscr{D}^{1 / 2,0}$ of the Lorentz group which is used in the description of a relativistic spin 1 particle. The components $(\mathbf{U}, W)$ correspond to the four-vector of the relativistic case which transforms under $\mathscr{D}^{1 / 2,1 / 2}$ and the components $(\mathbf{L}, \mathbf{M})$ to the antisymmetric tensor of rank two which transforms under $\mathscr{D}^{0,1 / 2} \oplus \mathscr{D}^{1 / 2,0}$.

\section{b) The Wave Equation}

From now on, we use the components $(\mathbf{U}, W ; \mathbf{L}, \mathbf{M})$ with the transformation properties (58) to describe the wave function of our spin 1 particle. These are in fact functions of space-time variables whose complete galilean transformation law is given by (48), with (58) giving the explicit form of the representation $\delta^{[2]}$ of the homogeneous Galilei group. The three independent components are given by the vector-valued wave function $\mathbf{U}$ which transforms according to (51) (with $N=2$ ), thereby illustrating the general theory of IV.b.

We now want to rewrite the Bargmann-Wigner equations with these components $(\mathbf{U}, W ; \mathbf{L}, \mathbf{M})$. We use, for that purpose, the definitions (57) and the Dirac-like equations (25) obeyed by $\left(\begin{array}{l}\varphi_{1} \\ \chi_{1}\end{array}\right)$ and $\left(\begin{array}{l}\varphi_{2} \\ \chi_{2}\end{array}\right)$. One obtains the following system:

$$
\left\{\begin{array} { l } 
{ \mathbf { L } = \mathbf { p } W - E \mathbf { U } } \\
{ \mathbf { M } = \mathbf { p } \times \mathbf { U } }
\end{array} \quad \left\{\begin{array}{l}
\mathbf{p} \cdot \mathbf{L}=m E W \\
\mathbf{p} \times \mathbf{M}=m \mathbf{L}-m E \mathbf{U}
\end{array} .\right.\right.
$$

From these ten equations for the ten components, one may deduce supplementary relations:

$$
\left\{\begin{array}{l}
\mathbf{p} \times \mathbf{L}=-E \mathbf{M} \\
\mathbf{p} \cdot \mathbf{M}=0
\end{array} \quad m W-\mathbf{p} \cdot \mathbf{U}=0\right.
$$

One checks that all these equations remain unchanged when $(\mathbf{C}, W ; \mathbf{L}, \mathbf{M})$ are subjected to the transformations $(58)$, along with $(E, \mathbf{p})$ being transformed according to the standard law:

$$
\left\{\begin{array}{l}
\mathbf{p}^{\prime}=R \mathbf{p}+m \mathbf{v} \\
E^{\prime}=E+\mathbf{v} \cdot R \mathbf{p}+\frac{1}{2} m \mathbf{v}^{2} .
\end{array}\right.
$$

This proves the Galilean invariance of our equations. Moreover, it follows from these equations that each one of the components $(\mathbf{U}, W ; \mathbf{L}, \mathbf{M})$ separately obeys the Schrödinger equation. In other words, the equations (59) viewed as an eigenvalue problem for the energy gives the expected Galilean value $E=\frac{\mathbf{p}^{2}}{2 m}$. 
In the same way as our equations (25) for spin $1 / 2$ appear as a nonrelativistic limit of the Dirac equation, the equations (59) may be derived by a suitable limit process from the Proca equations [20] which describe relativistic spin 1 particles.

\section{c) Electromagnetic properties}

The essential advantage of the wave equations (59) over the standard Galilei group representation for a $\operatorname{spin} s=1$, is to permitthe study of the particle behaviour in external fields. We introduce electromagnetic fields in the wave equation (59) by the minimal coupling recipe (10). The game consists in eliminating the auxiliary components $(W ; \mathbf{L}, \mathbf{M})$ to obtain an equation for the essential components $\mathbf{U}$. This equation will give the energy of the particle in presence of the fields. It is somewhat disappointing that this computation is a very tricky one, rather more involved than the corresponding elimination process in the relativistic case, where a covariant formulation is of a great help. We will not reproduce here these manipulations. Let us simply state the final results: the particle is endowed with a magnetic moment: $\boldsymbol{\mu}=\frac{q}{2 m} \mathbf{S}$, where $\mathbf{S}$ is its spin (i.e. the matrices $\left.\left(S^{k}\right)_{\alpha \beta}=i \varepsilon_{k \alpha \beta}\right)$. As in the spin $1 / 2$ case, this intrinsic magnetic moment is the same as the one obtained in RQM. But contrarily to the relativistic situation, our particle has no electric quadrupole moment. That this is a general property of galilean particles has been demonstrated in IV.c. Finally, there appear some derivative couplings between the electromagnetic field and the wave function (but such complicated terms also exist in the relativistic case).

\section{Galilean Electromagnetism}

a) The Wave Equations

The relativistic wave equations for a spin 1 massless particle are the Maxwell equations in vacuo:

$$
\left\{\begin{array} { r l } 
{ \mathbf { p } \cdot \mathscr { E } } & { = 0 } \\
{ \mathbf { p } \times \mathscr { H } } & { = E \mathscr { E } ( * ) }
\end{array} \quad \left\{\begin{array}{rl}
\mathbf{p} \times \mathscr{E}=-E \mathscr{H} \\
\mathbf{p} \cdot \mathscr{H}=0
\end{array}\right.\right.
$$

the fields $(\mathscr{E}, \mathscr{H})$ deriving from the potentials $(\mathscr{A}, \mathscr{V})$ :

$$
\left\{\begin{array}{l}
\mathscr{E}=\mathbf{p} \mathscr{V}-E \mathscr{A} \\
\mathscr{H}=\mathbf{p} \times \mathscr{A} .
\end{array}\right.
$$

Gauge transformations of the second kind:

$$
\left\{\begin{array}{l}
\mathscr{A} \rightarrow \mathscr{A}+\mathbf{p} \Lambda \\
\mathscr{V} \rightarrow \mathscr{V}+E \Lambda
\end{array}\right.
$$


where $\Lambda$ is some arbitrary scalar function, do not modify the fields.

What is now the situation in the Galilean case? Starting from our wave equations (59) and (60), setting $m=0$, and changing the notation $(\mathbf{U}, W ; \mathbf{L}, \mathbf{M})$ to the more conventional one $(\mathscr{A}, \mathscr{V} ; \mathscr{E}, \mathscr{H})$ as above, we obtain a similar system:

$$
\left\{\begin{array} { r l } 
{ \mathrm { p } \cdot \mathscr { E } } & { = 0 } \\
{ \mathrm { p } \times \mathscr { H } } & { = 0 ( * ) }
\end{array} \quad \left\{\begin{array}{l}
\mathrm{p} \times \mathscr{E}=-E \mathscr{H} \\
\mathbf{p} \cdot \mathscr{H}=0 .
\end{array}\right.\right.
$$

The definitions (63) of fields in terms of potentials remain valid as well as the form (64) of gauge transformations. Another way to derive the galilean wave-equation is to write Maxwell equations in the form:

$$
\left\{\begin{array} { c } 
{ \mathbf { p } \cdot \mathscr { E } = 0 } \\
{ \mathbf { p } \times \mathscr { H } = \frac { 1 } { c ^ { 2 } } E \mathscr { E } }
\end{array} \quad \left\{\begin{array}{l}
\mathbf{p} \times \mathscr{E}=-E \mathscr{H} \\
\mathbf{p} \cdot \mathscr{H}=0
\end{array}\right.\right.
$$

where we have introduced the constant $c$ (the velocity of light), implicitely taken as unity in the preceding formulas. In the nonrelativistic $c \rightarrow \infty$, one obviously obtains the same equations (65), although such a limit method in general may be misleading. Since Maxwell equations may be obtained from Proca equations by setting $m=0$, one may exchange the limits $m \rightarrow 0$ and $c \rightarrow \infty$, which checks the coherence of our results.

It is easily seen that the equations (65) imply:

$$
\Delta \mathscr{E}=0 \quad \Delta \mathscr{H}=0 .
$$

These equations also result directly from the relativistic equations

$$
\square \mathscr{E}=\Delta \mathscr{E}-\frac{1}{c^{2}} \frac{\partial^{2} \mathscr{E}}{\partial t^{2}}=0 \quad \square \mathscr{H}=0
$$

when taking the limit $c \rightarrow \infty$.

A natural Galilei-invariant gauge is fixed by the transcription to the present case, $m=0$, of the last of equations (60):

$$
\operatorname{div} \mathscr{A}=0
$$

(which is but the nonrelativistic limit of the Lorentz gauge condition). The potentials then also obey the equations:

$$
\Delta \mathscr{V}=0 \quad \Delta \mathscr{A}=0 .
$$

The fields $(\mathscr{A}, \mathscr{V} ; \mathscr{E}, \mathscr{H})$ describing a galilean spin 1 massless particle transform according to a representation of the Galilei group of the general form (44) with the peculiarity that the phase function $f$ now vanishes (see (8)). This corresponds to the fact that galilean particles with zero mass belong to true (vector) representations of the Galilei group [7] contrarily to nonzero mass particles which correspond to nontrivial ray representations. We still have to exhibit the representation of the homo- 
geneous Galilei group. It is given by formulas (58) where one sets $m=0$. The representation now decomposes, the potentials $(\mathscr{A}, \mathscr{V})$ on the one hand, the fields $(\mathscr{E}, \mathscr{H})$ on the other separately transforming according to:

$$
\begin{aligned}
& \left\{\begin{array}{l}
\mathscr{A}^{\prime}=R \mathscr{A} \\
\mathscr{V}^{\prime}=\mathscr{V}+\mathrm{v} \cdot R \mathscr{A}
\end{array}\right. \\
& \left\{\begin{array}{l}
\mathscr{E}^{\prime}=R \mathscr{E}+\mathrm{v} \times R \mathscr{H} \\
\mathscr{H}^{\prime}=R \mathscr{H} .
\end{array}\right.
\end{aligned}
$$

It suffices to evaluate the left hand sides at the point $\left(\mathrm{x}^{\prime}, t^{\prime}\right)$ transformed from the argument $(x, t)$ of the right hand side by the considered Galilei transformation, to obtain the complete relevant representation of the Galilei group. Equations (65) and (63) are invariant when the wave function components are subjected to the transformation (71), while the energy momentum operators $(E, \mathbf{p})$ transform according to:

$$
\left\{\begin{array}{l}
\mathbf{p}^{\prime}=R \mathrm{p} \\
E^{\prime}=E+\mathrm{v} \cdot R \mathrm{p}
\end{array}\right.
$$

since we consider a zero mass case (compare (61)).

\section{b) Physical Discussion}

When comparing Maxwell equations (62) with their nonrelativistic counterparts, eq. (65), we see that only the equations labelled (*) differ. The curl of the magnetic field, vanishing in the nonrelativistic approximation, in fact is given by the famous "displacement current" introduced by Maxwell and which gives all their importance to his equations. The absence of this term in the galilean case means that the Maxwell equations introduce relativity (under the Poincaré group) in a quite literal sense. In physical situations where the displacement current is of negligible importance, the predictions of the theory are in perfect agreement with galilean relativity. The whole of pre-Maxwellian electromagnetism (laws of Faraday, Ampère, Biot-Savart etc. ...) is simultaneously exact and consistent with the old Newtonian conception of space-time. But as soon as one takes into consideration the essentially Maxwellian equation (62), one obtains specifically relativistic phenomena such as the propagation of electromagnetic waves with constant velocity etc. This is the one equation which definitely ruins the old Galilean relativity, introducing the Einsteinian one. For an important class of phenomena, however, equations (62) and (65) coincide: such is the case if one is interested in static, time independent, electromagnetic fields ${ }^{9}$,

${ }^{9}$ More generally one could consider magnetic fields with a linear time dependence and time independent electric fields. But this case does not present any practical interest. 
for which the displacement current obviously vanishes. This proves that we do not risk any internal contradiction when introducing static electromagnetic fields in a nonrelativistic theory, the galilean invariance of the theory being unaffected. These considerations also provide a justification for the use of gauge invariance of the second kind (we have shown the expressions (65) of gauge invariance to be consistent with galilean invariance) in nonrelativistic wave equations, provided we only consider static fields. This is a very important case in practice since it suffices for studying Coulomb interactions as well as the electric and magnetic multipole moments of a system. Of course, there is also a wide class of phenomena where the nonrelativistic equations remain approximately valid, even with time dependent electromagnetic fields (low frequency phenomena, etc....).

The only monochromatic plane-wave solutions of eq. (65) are of the following two rather trivial types:

$$
\text { a) }\left\{\begin{array} { r l } 
{ \mathscr { E } } & { = \mathscr { E } _ { 0 } = C ^ { t e } } \\
{ \mathscr { H } } & { = \mathscr { H } _ { 0 } = C ^ { t e } }
\end{array} \quad \text { b) } \left\{\begin{array}{rl}
\mathscr{E} & =\mathscr{E}_{0} e^{-i \omega t} \\
\mathscr{H} & =0
\end{array}\right.\right.
$$

so that there is no propagation, although one might interpret b) as an electric field propagating with infinite velocity, which is consistent with the intuitive meaning of a nonrelativistic limit as corresponding to $c \rightarrow \infty$. Case b) also corresponds to the so-called "dipole approximation" in semi-classical radiation theory, thus shown to be compatible with galilean invariance ${ }^{10}$.

Equations (65) can be used to discuss galilean electromagnetic fields, not only free ones, but also in presence of sources. It suffices to add charge and current densities in the right-hand side of the first pair of eq. (65), as in the Maxwellian case. However, the field equations no longer imply local conservation of the electric charge. After all, such a local conservation law, insured by the displacement current in Maxwell equations, certainly is a specific relativistic requirement, as shown by the standard Einstein argument based on the relativity of simultaneity.

Considering now a classical (non-quantum, nonrelativistic) particle in a galilean electromagnetic field which obeys the equations (66), it is seen that the usual Hamiltonian:

$$
H=\frac{1}{2 m}(\mathbf{p}-q \mathscr{A})^{2}+q \mathscr{V}
$$

leads to the equation of motion:

$$
m \ddot{\mathbf{r}}=q(\mathscr{E}+\dot{\mathbf{r}} \times \mathscr{H})
$$

10 A concrete application [22] of these ideas is a consistent nonrelativistic description of the behaviour of electrons in intense electromagnetic waves (laser beams) which exhibits in a very simple way some of the peculiar features usually derived from involved relativistic considerations [23]. 
so that the well-known expression of the Lorentz force retains its validity in nonrelativistic situations. This is an old result, implicitly used in many places, but whose internal consistency it is perhaps useful to emphasize here.

As to the energy carried by the electromagnetic field itself, considerations similar to those of the relativistic case, when applied to eq. (65), show that the energy density of the field must be defined by:

$$
W=\frac{1}{8 \pi} \mathscr{H}^{2}
$$

which is quite in keeping with the relativistic expression $W$ $=\frac{1}{8 \pi}\left(\mathscr{H}^{2}+\frac{1}{c^{2}} \mathscr{E}^{2}\right)$, in the limit $c \rightarrow \infty$. The Poynting vector giving the flux density of the field energy retains its expression:

$$
\mathrm{S}=\frac{1}{4 \pi} \mathscr{E} \times \mathscr{H}
$$

One might be somewhat puzzled by the fact that, in this galilean electromagnetism, an electric field does not carry any energy according to (76). But this completely agrees with the absence of the displacement current in (65). As a matter of fact, it is the displacement current which makes possible the existence of electric currents in circuits that are not complete loops, in other words the transport of energy by means of electric fields. The working of capacitors in a.c. circuits for instance, results from the specifically relativistic features of electromagnetism.

It is commonly stated that electric fields and magnetic fields are two separate entities in nonrelativistic theory, mixing only under the effect of Lorentz transformations, which make them to appear as two aspects of a same fundamental quantity, the electromagnetic field. The galilean transformation properties (71) show the necessity of revising this opinion: if a "pure" electric field may be defined in an absolute manner, independent of the frame of reference, this is not the case for the magnetic field. A magnetic field which is pure in a certain frame, gives rise to a certain electric field component in another frame, uniformly moving with respect to the first one ${ }^{11}$. Indeed this is the standard effect accounting for the appearance of an electromotive force in a conductor moving through a magnetic field, the expression for which could be easily deduced from the relevant equation (71). This effect, on which is founded most of our electrical technology, is thus shown not to be specifically relativistic.

A last remark about these galilean transformation properties of "nonrelativistic electromagnetic fields" may be in order: there is a close connection between the fact that a pure electric field remains such in

11 This has also been noted by Havas in ref. [24], footnote 29. 
any galilean frame on one hand and the absence of spin-orbit coupling in nonrelativistic wave equations (see the case of spin $1 / 2$, III.d) on the other hand. In RQM, spin-orbit coupling, which appears for instance in the Dirac equation for an electron in the nuclear electric field, may be viewed as resulting from two simultaneous effects: i) a magnetic field is generated in the rest system of the electron by its motion through the nuclear Coulomb field, and ii) one must take into account the so-called Thomas precession of the spin. This second effect is well-known to be a purely relativistic one; what we wish to emphasize is that the same is true of the first effect, so that it is not consistent to consider them separately or to talk of the Thomas precession as a "relativistic correction".

\section{Summary and Conclusions}

In this paper we have considered the description of nonrelativistic particles, in order to compure their properties to those of the corresponding relativistic particles, thus obtaining a clearcut distinction between the specifically relativistic features of the theory and those which equally follow from a consistent nonrelativistic treatment.

Nonrelativistic particles, defined through the theory of unitary irreducible representations of the Galilei group (invariance group of nonrelativistic physics), are characterized, exactly as in the nonrelativistic case, by their mass and spin-spin in particular is thus seen neither to "result" from some relativistic wave equation, nor to be a purely relativistic effect. The relevant Galilei group representation gives complete information on the free particle behaviour, describing all of its kinematical properties.

In order to study the dynamical properties of the particle, however, it is necessary to describe it by means of a wave equation which enables us to discuss the effect of external fields on the system. We have been able to construct a theory of nonrelativistic wave equations for any spin, based on the theory of representations of the Galilei group.

Our scheme is founded upon the properties of a new nonrelativistic wave equation for a spin $1 / 2$ particle which we derived as did DIRac in the relativistic case. This equation implies the Pauli equation with the correct value of the electron gyromagnetic ratio, so that one does not either deal here with a relativistic effect.

However, using very simple dimensional arguments, it has been shown that nonrelativistic elementary particles could not possess higher order multipole moments besides an electric charge and a magnetic dipole moment, in contradistinction to the relativistic case.

We also studied in detail "galilean electromagnetism", thereby demonstrating which feature of the Maxwell equations requires Galilean 
relativity to be replaced by the Einsteinian one: it is precisely the presence of the very "displacement current" introduced by Maxwell. Interesting conclusions may be drawn from the Galilean transformation law of the electromagnetic field.

Finally, let us emphasize that some puzzling questions occur when we compare the connection between representations of the kinematical group and associated wave equations in RQM and NQM. There seem to be open problems about the notion of wave equation (necessity to introduce redundant components for the wave functions, intervening of the homogeneous Lorentz - or Galilei - group, etc. . .). We have suggested [1] that a detailed analysis of gauge invariance of the second kind and its relations with the relativity group could perhaps clarify the situation. It might also hopefully give an easier and quickier way to study the electromagnetic properties of particles.

Acknowledgements. This work has greatly benefitted from the continuous interest of F. Lurçat. Stimulating discussions with G. Flamand and M. LÉvyNAHAS are gratefully acknowledged.

\section{References}

1. Lévy-Leblond, J.-M.: Thèse de Doctorat, Orsay, 1965.

2. Wrgner, E. P.: Ann. Math. 40, 149 (1939).

3. JAUCH, J. M.: Helv. Phys. Acta 37, 284 (1964).

4. Bargmanx, V.: Ann. Math. 59, 1 (1954).

5. Trönu, E., and E. P. Wigner: Nuovo Cimento 9, 705 (1952).

6. Hanermeshe, H.: Ann. Phys. (N.Y.) 9, 518 (1960).

7. LÉvy-Leblond, J.-M.: J. Math. Phys. 4, 776 (1963).

8. - Commun. Math. Phys. 4, 157 (1967).

9. Newton, T. D., and E. P. Wigner: Rev. Mod. Phys. 21, 400 (1956) - WightMaN, A. S.: Rev. Mod. Phys. 34, 845 (1962).

10. Dirac, P. A. M.: Proc. Roy. Soc. A 117, 610 (1928).

11. Pauli, W.: Z. Physik 43, 601 (1927).

12. Galindo, A., and C. Sanchez del Rio: Am. J. Phys. 29, 582 (1961).

13. Eberlein, W. F.: Am. Math. Monthly 69, 587 (1962).

14. Pursey, D. L.: Ann. Phys. (N.Y.) 32, 157 (1965).

15. Gel'fand, I. M., R. A. Mrnlos, and Z. Y. Shapiro: Representations of the rotation and Lorentz groups. Oxford: Pergamon Press 1963.

16. Georges, C., and M. Lévy-Nahas: J. Math. Phys. 7, 980 (1966).

17. Ixönv, E., and E. P. Wigner: Proc. Nat. Acad. Sci. 39, 510 (1953); 40, 119 (1954). - Saletan, E.: J. Math. Phys. 2, 1 (1961).

18. Bargmand, V., and E. P. Wigner: Proc. Nat. Acad. Sci. 34, 211 (1948).

19. Moldauer, P. A., and K. M. Case: Phys. Rev. 102, 279 (1956).

20. Proca, A.: C. R. Acad. Sci. 202, 1420 (1936); J. Phys. Radium 9, 61 (1938).

21. Tumanov, V. S.: Soviet Physics JETP 19, 1182 (1964).

22. LÉvy-Leblond, J.-M.: Communication at the Second Rochester Conference on Coherence and Quantum Optics. Rochester: 1966.

23. Kibble, T. W.: Phys. Rev. 150, 1060 (1966).

24. Havas, P.: Rev. Mod. Phys. 36, 938 (1964). 